\title{
ON THE FOUNDATIONS OF INVERSE MONOIDS AND INVERSE ALGEBRAS
}

\author{
by JONATHAN LEECH
}

(Received 18th October 1995)

\begin{abstract}
Symmetric inverse monoids of objects in arbitrary categories are studied. Necessary and sufficient conditions are given for such monoids to be E-unitary or else form (complete) inverse algebras. Particular attention is given to symmetric inverse monoids of objects in free categories.
\end{abstract}

1991 Mathematics subject classification: $20 \mathrm{M} 18$.

\section{Introduction}

The primary motivation for studying inverse semigroups has been that they are the algebraic structures encountered most of ten in the formal study of partial symmetries. Yet numerous significant (types of) mathematical objects have partial symmetries which collectively possess a richer algebraic structure than that captured in the definition of an inverse semigroup. This observation has led the author to define an "inverse algebra" to be any inverse monoid $S$ possessing a natural meet operation. Thus given any pair of elements $x, y \in S$, an infimum $x \wedge y$ exists relative to the natural partial ordering. The algebra is called "complete" when arbitrary nonempty subsets possess natural infima. A primarily algebraic investigation was carried out on both types of algebras by the author in [13].

We shall refer to inverse semigroups of partial symmetries as symmetric inverse monoids. This is an extension of the usual meaning of the term symmetric inverse monoid which usually refers to the inverse monoid of all partial bijections on a set.

If symmetric inverse monoids turn out to be complete inverse algebras in so many instances where the corresponding objects are of mathematical interest, then why is this so? This leads us to consider a related, but more precisely posed question: When are the symmetric inverse monoids for all objects in a given category necessarily (complete) inverse algebras? It is the purpose of this paper to provide answers to this and several closely related questions.

Since these answers are stated in the terminology of category theory, we begin by recalling in Section 1 the description of symmetric inverse monoids in abstract categories in [12], and then stating precise conditions for a given category to have symmetric inverse monoids existing at each of its objects. The essential agreement of this description with the familiar "isomorphisms between subobjects" picture of a 
symmetric inverse monoid is demonstrated, and examples are given showing how to exploit instances of duality between categories.

Section 2 begins by first recalling the definition of an inverse algebra and then examining an alternative description not found in [13]. The above question about when the symmetric inverse monoids of all objects in a given category must be (complete) inverse algebras is then considered, with precise answers given in Theorems 2.6 and 2.7. Criteria insuring that symmetric inverse monoids in a given category are respectively E-unitary monoids, finitely complete inverse algebras or semilattices, are given in Theorems 2.8, 2.10 and 2.16.

In the third and final section, symmetric inverse monoids of objects in free categories are studied. In so doing, certain inverse monoids arising in the study of $\omega$-semigroups are cast in a new light.

\section{Settings and inverse monoids}

A monocontext is a pair $[\mathbf{M}, X]$ consisting of a category $\mathbf{M}$ whose morphisms are all monic and an object $X$, called the maximal object of $\mathbf{M}$, such that every object of $\mathbf{M}$ is a subobject of $X$. Upon dualizing one has the concept of an epicontext $(E, X)$ where $\mathbf{E}$ is a category of epimorphisms, whose objects are all quotient objects of the maximal object $\boldsymbol{X}$. One visualizes a monocontext as a "flow" of monomorphisms moving towards the maximal object, while an epicontext is visualized in reverse fashion. $(M, X)$ is called a monosetting when $M$ also has finite intersections. An episetting $(\mathbf{E}, X)$ is defined in dual fashion. Often the terms "context" or "setting" will be used when we do not wish to specify whether the situation is monic or epic.

An equivalence of contexts is any equivalence functor between the ambient categories which preserves the maximal objects. A duality of contexts is a duality (contravariant equivalence) between the ambient categories preserving the maximal objects. Given an equivalence or a duality of contexts, if either context is a setting, then so is the other. Contexts and settings are encountered in ordinary categories as follows:

Let $\mathbf{K}$ be a category and let $X$ be any object in $\mathbf{K}$. The monocontext of $X$ in $\mathbf{K}$ is the pair $(\mathbf{M}(X), X)$, where $\mathbf{M}(X)$ denotes the subcategory of $\mathbf{K}$ of all monomorphisms between all objects $A$ of $\mathbf{K}$ for which a monomorphism $\alpha: A \rightarrow X$ exists. One defines in dual fashion the epicontext $(\mathrm{E}(X), X)$ of $X$ in $\mathbf{K}$. When $\mathbf{K}$ has finite [co-] intersections, then all monocontexts, [respectively, epicontexts] in $\mathbf{K}$ are monosettings [episettings]. Any equivalence [or duality] between a pair of categories induces a host of offspring equivalences [dualities] between the various derived contexts in the two categories.

From any small setting one can construct an inverse monoid of fractional morphisms which classifies that setting to within (contravariant) equivalence. This directly generalizes results published in 1953 by A. H. Clifford [3] who showed that any bisimple inverse monoid $S$ can be constructed from a one object episetting, that is, from a right cancellative monoid whose principal left ideals are closed under finite intersection. We present the construction for monosettings.

Let $(M, X)$ be a small monosetting. Consider parallel pairs of morphisms $\left(\alpha, \alpha^{\prime}\right): A \rightarrow X$ in $\mathbf{M}$ where the common domain $A$ varies with the pair. Two such pairs 
$\left(\alpha, \alpha^{\prime}\right)$ and $\left(\beta, \beta^{\prime}\right)$ are equivalent if there is an isomorphism $\mu \in \mathbf{M}$ such that both $\beta=\alpha \mu$ and $\beta^{\prime}=\alpha^{\prime} \mu$. A fractional morphism is an equivalence class of such pairs, with the class of $\left(\alpha, \alpha^{\prime}\right)$ denoted by $\left[\alpha, \alpha^{\prime}\right]$. Let $\mathrm{I}(\mathbf{M}, X)$ denote the set of all such fractions. A multiplication is defined on $\mathbf{I}(\mathbf{M}, X)$ by setting

$$
\left[\alpha, \alpha^{\prime}\right]\left[\beta, \beta^{\prime}\right]=\left[\alpha \kappa, \beta^{\prime} \lambda\right]
$$

where $\kappa$ and $\lambda$ arise from taking the intersection of the middle pair, $\alpha^{\prime}$ and $\beta$, in $\mathbf{M}$ : $\alpha^{\prime} \cap \beta=\alpha^{\prime} \kappa=\beta \lambda$. It is easy to see that the class $\left[\alpha \kappa, \beta^{\prime} \lambda\right]$ is independent (i) of the particular choice of $(\kappa, \lambda)$ to represent the intersection $\alpha^{\prime} \cap \beta$, and (ii) of the particular representatives, $\left(\alpha, \alpha^{\prime}\right)$ and $\left(\beta, \beta^{\prime}\right)$. Multiplication is thus well defined. We have the following pair of results taken from [12]. (See also [5, Section VII.8].)

Theorem 1.1. Given a small monosetting $(\mathbf{M}, X), \mathrm{I}(\mathbf{M}, X)$ forms an inverse monoid under the above multiplication. In particular:

(i) the idempotents of $\mathrm{I}(\mathrm{M}, X)$ are classes of the form $[\alpha, \alpha]$, with the identity element being $\left[1_{X}, 1_{X}\right]$.

(ii) the semilattice of idempotents in $\mathrm{I}(\mathrm{M}, X)$ is isomorphic with the semilattice of subobjects of $X$.

(iii) inverses in $\mathrm{I}(\mathbf{M}, X)$ are given $b$ the rule, $\left[\alpha, \alpha^{\prime}\right]^{-1}=\left[\alpha^{\prime}, \alpha\right]$.

Theorem 1.2. Two small monosettings are equivalent if and only if their classifying monoids are isomorphic. In particular, given an equivalence of small monosettings, $\mathrm{F}:(\mathrm{M}, X) \approx(\mathrm{N}, Y)$, an isomorphism $\mathrm{I}_{\mathrm{F}}: \mathrm{I}(\mathrm{M}, X) \cong \mathrm{I}(\mathrm{N}, Y)$ is defined by $\mathrm{I}_{\mathrm{F}}\left[\alpha, \alpha^{\prime}\right]=$ $\left[\mathrm{F} \alpha, \mathrm{F} \alpha^{\prime}\right]$. Moreover, to within isomorphism, every inverse monoid arises as the classifying monoid for some small monosetting.

The inverse monoid $\mathrm{I}(\mathbf{M}, X)$ is thus called the classifying monoid of the monosetting $(M, X)$. In this paper we only need the obvious assertion that the induced map $I_{F}$ is an isomorphism; however, the reader should take note of the final assertion of Theorem 1.2 which guarantees that the scope of this paper is all inverse monoids and inverse algebras.

Upon dualizing the above concepts, any small episetting $(E, X)$ has a classifying monoid I(E, $X)$ defined on the set of equivalence classes $\left[\alpha, \alpha^{\prime}\right]$ of pairs $\left(\alpha, \alpha^{\prime}\right)$ of epimorphisms form $X$ to some common codomain. Multiplication is given by cointersection in the middle: $\left[\alpha, \alpha^{\prime}\right]\left[\beta, \beta^{\prime}\right]=\left[\kappa \alpha, \lambda \beta^{\prime}\right]$, where $\kappa \alpha^{\prime}=\lambda \beta$ is the co-intersection of $\alpha^{\prime}$ with $\beta$. Clearly $\mathrm{I}(\mathbf{E}, X)$ is an inverse monoid and the dual assertions of Theorems 1.1 and 1.2 must hold.

Let $\mathbf{K}$ be a well-powered (i.e., locally small) category with object $X$. If the context $\mathbf{M}(X)$ is a setting, then the symmetric inverse monoid of $X$ in $\mathbf{K}$, denoted $I_{X}$, is the classifying monoid of any small monosetting $(M, X)$ equivalent to $(M(X), X)$. Since any two choices for $(M, X)$ must be equivalent, $I_{X}$ is well defined to within isomorphism. For objects $X$ in most categories of mathematical interest, there is a 
standard choice for $\mathbf{M}(X)$ and thus a standard form for $I_{X}$. Suppose instead that the duals to these conditions all hold at $X$. In this case, the dual symmetric inverse monoid $I_{X}^{*}$ at $X$ is defined as the classifying monoid of any small episetting $(\mathbf{E}, X)$ equivalent to $(\mathbf{E}(X), X)$. Again $I_{X}^{*}$ is unique to within isomorphism. Most important categories in mathematics have both types of monoids existing for each of their objects, with the two monoids usually being non-isomorphic. In any case, a category has [dual] symmetric inverse monoids if such monoids exist at each of its objects. We sum up our remarks with the following theorem.

Theorem 1.3. A well-powered category has symmetric inverse monoids if and only if it has finite intersections. Dually a co-well-powered category has dual symmetric inverse monoids if and only if it has finite co-intersections.

The essential agreement of our description of symmetric inverse monoids with the standard description in terms of isomorphisms between subobjects of the ambient object (the groupoid approach) is demonstrated as follows.

Example 1.4. Let $X$ be a set in the category Set of sets and functions; let $\left[\alpha, \alpha^{\prime}\right]$ be a fractional morphism in $I_{X}$, with $A, A^{\prime} \subseteq X$ being the respective images of $\alpha$ and $\alpha^{\prime}$ in $X$. Both $A$ and $A^{\prime}$ are independent of the pair $\left(\alpha, \alpha^{\prime}\right): D \rightarrow X$ representing $\left[\alpha, \alpha^{\prime}\right]$. Let $\alpha$ and $\alpha^{\prime}$ be factored as $\alpha=\imath \delta$ and $\alpha^{\prime}=\imath^{\prime} \delta^{\prime}$, where $\imath$ and $\imath^{\prime}$ are the inclusions of $A$ and $A^{\prime}$ into $X$, and $\delta: D \rightarrow A^{\prime}$ are bijections. Then the bijection $\sigma=\delta\left(\delta^{\prime}\right)^{-1}: A^{\prime} \rightarrow A$ also depends only on the class $\left[\alpha, \alpha^{\prime}\right]$. Thus: Elements of $I_{X}$ are uniquely represented by bijections between subsets of $X$ under the compound bijection $\sigma \rightarrow\left(\imath \sigma, \imath^{\prime}\right) \rightarrow\left[\imath \sigma, \imath^{\prime}\right]$, where $\sigma: A^{\prime} \rightarrow A$ is such a bijection, with $t$ and $l^{\prime}$ being inclusion maps of $A$ and $A^{\prime}$ into $X$.

Let $\left[\beta, \beta^{\prime}\right] \in I_{X}$ be represented by $\left(\eta \tau, \eta^{\prime}\right)$, for $\tau: B^{\prime} \rightarrow B$ a bijection between the images $B$ and $B^{\prime}$ in $X$ of $\beta^{\prime}$ and $\beta$, with the $\eta^{\prime}$ 's being the inclusion maps for these images. We compute $\left[\alpha, \alpha^{\prime}\right]\left[\beta, \beta^{\prime}\right]$ using $\left(\imath \sigma, \imath^{\prime}\right)$ and $\left(\eta \tau, \eta^{\prime}\right)$. The intersection $\imath^{\prime} \cap \eta \tau$ is obtained from the subset $C=\tau^{-1}\left(A^{\prime} \cap B\right)$ of $B^{\prime}$ together with the restriction $\kappa=\tau \mid C: C \rightarrow A^{\prime}$ and the inclusion $\lambda: C \subseteq B^{\prime}$.

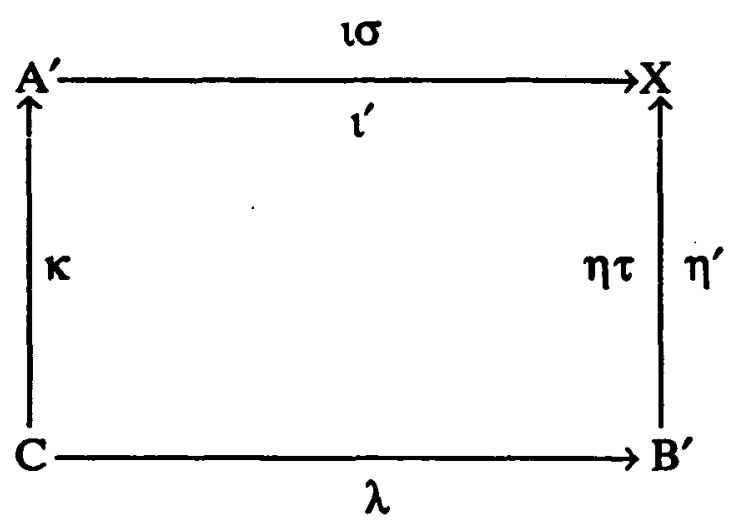


The product is precisely the class of $\left(l \sigma \kappa, \eta^{\prime} \lambda\right)$ where $\eta^{\prime} \lambda$ is just the inclusion of $C$ into $X$. Upon calculating the bijection representing this class we obtain the composition $\left(\sigma \mid A^{\prime} \cap B\right)(\tau \mid C)$. Hence, for Set, our definition of $I_{X}$ is equivalent to the familiar one.

The details of the above example easily extend to any category of concrete mathematical objects. Indeed they may be generalized to objects $X$ in any wellpowered category $\mathbf{K}$ having finite intersections. Upon choosing for each equivalence class of subobjects of $X$ a representative monomorphism (the designated inclusion) $\imath: A \rightarrow X$, every fraction $\left[\alpha, \alpha^{\prime}\right]$ is uniquely represented by a pair $\left(\imath \sigma, \imath^{\prime}\right)$ where $\imath$ and $\imath^{\prime}$ both represent their subobject classes and $\sigma: A \rightarrow A^{\prime}$ is an isomorphism between their domains. Because $K$ has finite intersections, the multiplication of fractions $\left[\alpha, \alpha^{\prime}\right]\left[\beta, \beta^{\prime}\right]$ may again be carried out by composing isomorphisms between subobject representatives.

The monoid-of-fractions approach facilitates the use of instances of duality between categories to double the fruit of our labours.

Lemma 1.5. Let $\mathbf{F}: \mathbf{K} \rightarrow \mathbf{K}^{\prime}$ be a duality functor. Upon restriction, $\mathbf{F}$ induces for each object $X$ in $\mathrm{K}$ a duality of $(\mathrm{M}(X), X)$ with $(\mathrm{E}(\mathrm{F} X), \mathrm{F} X)$ and similarly, a duality of $(\mathbf{E}(X), X)$ with $(\mathbf{M}(\mathrm{F} X), \mathrm{F} X)$.

Theorem 1.6. Let $\mathrm{F}: \mathbf{K} \rightarrow \mathbf{K}^{\prime}$ be a duality functor. If $\mathbf{K}$ has symmetric inverse monoids, then $\mathbf{K}^{\prime}$ has dual symmetric inverse monoids. Moreover at each object $X$ in $\mathrm{K}, \mathrm{F}$ induces an isomorphism of $I_{X}$ with $I_{\mathrm{FX}}^{*}$.

Proof. Let $(M, X)$ be a small subcontext of $(M(X), X)$ for which inclusion is an equivalence. Then $F$ induces, by restriction, a duality of $(M, X)$ with (FM, $F X)$, with the inclusion of the latter into $(\mathrm{E}(\mathrm{FX}), \mathrm{FX})$ being an equivalence. $(\mathbf{M}(X), X)$ is thus a setting if and only if $(\mathrm{E}(\mathrm{F} X), \mathrm{F} X)$ is likewise, in which case both $I_{X}$ and $\Gamma_{\mathrm{F} X}^{*}$ exist. If the two monoids are constructed from the restricted settings $(M, X)$ and $(F M, F X)$, then the map $\left[\alpha, \alpha^{\prime}\right] \rightarrow\left[F \alpha, F \alpha^{\prime}\right]$ yields an isomorphism of classifying monoids.

Examples 1.7. Some familiar instances of duality may be applied as follows.

(i) Pontryagin duality for locally compact Hausdorff Abelian groups restricts to yield a duality between the category of discrete groups and the category of compact, Hausdorff abelian groups. Thus if $A$ is an abelian group and $A^{*}$ denotes its compact character group, then both $I_{A} \cong I_{A^{*}}$ and $I_{A^{*}} \cong I_{A^{*}}$.

(ii) In particular, since $A \cong A^{*}$ for finite abelian groups, it follows that in the category of abelian groups, the symmetric inverse monoid of a finite abelian group is isomorphic with its dual symmetric inverse monoid.

(iii) If $V^{*}=\operatorname{Hom}(V, F)$ is the dual space of a vector space $V$ over field $F$, then one has $V \cong V^{*}$ in the finite dimensional case as both dimensions agree. It follows that the symmetric inverse monoid of any finite dimensional vector space $V$ is isomorphic with its dual symmetric inverse monoid. 
Example 1.8. We calculate simultaneously $I_{Z}$ and $I_{T}^{*}$ where $T$ is the multiplicative circle group $\{z \in \mathbf{C}|| z \mid=1\}$ forming the Pontryagin dual of the additive group of integers, $\mathbf{Z}$. To begin, let $\mathbf{P}$ be the inverse monoid having as elements pairs $\langle m, n\rangle$ of positive integers, with the multiplication defined by:

$$
\langle m, n\rangle\langle p, q\rangle=\langle m p / \operatorname{gcd}\{n, p\}, n q / \operatorname{gcd}\{n, p\}\rangle
$$

where $\operatorname{gcd}\{n, p\}$ denotes the greatest common divisor of $n$ and $p . \mathbf{P}$ is the multiplicative analogue of the bicyclic semigroup $\mathbf{B}$ defined on $\mathbf{N} \times \mathbf{N}$ (for $\mathbf{N}$ denoting the natural numbers) with bicyclic "addition" given by

$$
(m, n)+(p, q)=(m+p-\min \{n, p\}, n+q-\min \{n, p\}) .
$$

Prime power factorization yields an isomorphism of $\mathbf{P}$ with the "direct sum" $\Sigma^{\omega} \mathbf{B}$, consisting of all $\omega$-tuples $\left\langle\left(u_{k}, v_{k}\right)\right\rangle \in \mathbf{B}^{\omega}$ such that $\left(u_{k}, v_{k}\right)=(0,0)$ for all but finitely many $\mathbf{k}$. The isomorphism is defined by

$$
\left.\langle m, n\rangle \rightarrow\left\langle\left(\log _{p} m, \log _{p} n\right)\right| p \text { is the } k \text {-th prime }\right\rangle
$$

where $\log _{p}$ denotes the $p$-exponent in the prime power factorization of the argument. Next let sgn $=\{+,-\}$ be the "sign group" with multiplication given by the usual rule of signs. Claim: $I_{Z}$ and $I_{T}^{*}$ are both isomorphic with $(\mathbf{s g n} \times \mathbf{P})^{0}$. To see this, consider the skeletal monosetting at $Z$ consisting of objects, $0=\{0\}$ and $Z$, together with morphisms: $1_{0}$ at $0 ; 0: 0 \rightarrow Z$; and $n: Z \rightarrow Z$ for all $n \neq 0$ in $Z$ where $n$ here represents the monomorphism $z \rightarrow n z$ for $z \in \mathbf{Z}$. Elements of the classifying monoid consist of $[0,0]$ and all $[m, n]$ such that $m, n \neq 0$, with $[m, n]$ being identified with $[-m,-n]$. Due to this identification, when forming a product $[m, n][p, q]$ we may assume $n, p \geq 0$. Case $1:$ if $[m, n]$ or $[p, q]$ is $[0,0]$, then $n$ and $p$ intersect at 0 and so the product is $[0,0]$. Case 2: neither are $[0,0]$. Then one has the intersection diagram

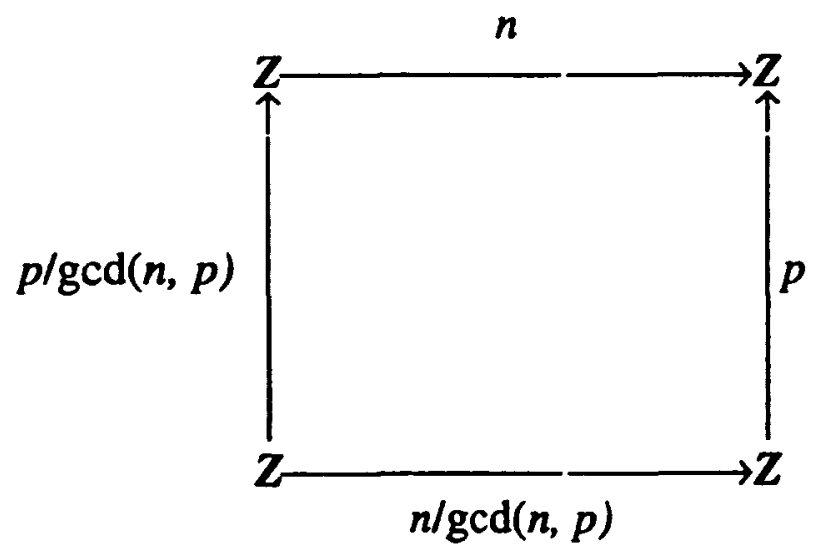


which yields $[m, n][p, q]=[m p / \operatorname{gcd}(n, p), n q / \operatorname{gcd}(n, p)]$. Let us denote $[m, n]$ by $\langle|m|,|n|\rangle$ when $m$ and $n$ share a common sign, and by $-\langle|m|,|n|\rangle$ when the signs are opposite. With this notation, the claim follows.

Example 1.9. Consider the additive group of real numbers $\mathbf{R}$ endowed with its standard topology. $\mathbf{R}$ is isomorphic to its Pontryagin dual (in the category of locally compact Hausdorff Abelian groups) $\mathbf{R}^{*}$ under the mapping $x \rightarrow x^{*}$ where $x^{*}(y)=\cos (x y)+i \sin (x y)$. The monosetting at $\mathbf{R}$ is thus dual to its episetting, so that $I_{\mathrm{R}}$ and $I_{\mathrm{R}}^{*}$ are isomorphic.

The structure of $I_{R}$ is nontrivial. Indeed let $\mathbf{R}_{\mathrm{D}}$ be the additive group of real numbers with the discrete topology. Then the identity map $\mathbf{R}_{\mathbf{D}} \rightarrow \mathbf{R}$ is a continuous homomorphism which yields, in turn, the inverse monoid $I_{R_{D}}$ as a subsemigroup (in fact, the maximal ideal) of $I_{R}$.

Thus far we have restricted our attention to the subcategory $\mathbf{M}(\mathbf{K})$ of all monomorphisms and the subcategory $\mathbf{E}(\mathbf{K})$ of all epimorphisms of some given category $\mathbf{K}$. Other subcategories may also be of interest, especially in concrete situations where monomorphisms need not be injective or epimorphisms need not be surjective. In such cases, there is the subcategory $\mathbf{I n j}(\mathbf{K})$ of injective monomorphisms and the subcategory Sur(K) of surjective epimorphisms. There is also the class $\mathbf{M}^{\mathbf{x}}(\mathbf{K})$ of extremal monomorphisms and the class $\mathbf{E}^{\mathbf{x}}(\mathbf{K})$ of extremal epimorphisms. (Recall that a monomorphism $\mu$ is called extremal, if given a factorization $\mu=v \varepsilon$ where $\varepsilon$ is epic, then $\varepsilon$ is an isomorphism. Extremal epimorphisms are defined in dual fashion. Regular monomorphisms which arise as equalizers are always extremal.) In general, neither $\mathbf{M}^{\mathbf{x}}(\mathbf{K})$ nor $\mathbf{E}^{\mathbf{x}}(\mathbf{K})$ need be subcategories of $\mathbf{K}$, but they are in many cases, and in particular when they coincide with $\mathbf{I n j}(\mathbf{K})$ or $\operatorname{Sur}(\mathbf{K})$ respectively. For any of these variant forms of $\mathbf{M}(\mathbf{K})$ or $\mathbf{E}(\mathbf{K})$, the corresponding statement of Theorem 1.3 or its dual still hold.

Example 1.10. Let $X_{\mathrm{R}}$ denote the inverse monoid of isomorphisms between the extremal subobjects of $\mathbf{R}$, the additive reals with the usual topology. (We are thus considering $I_{R}$ relative to $\mathbf{M}^{\mathbf{x}}(K)$ for the category $K$ of locally compact Hausdorff Abelian groups.) To within isomorphism, the extremal subobjects of $\mathbf{R}$ consist of all closed subgroups of $\mathbf{R}$ with the inherited topology: $\mathbf{R}$ and its cyclic subgroups, $\mathbf{Z} x$ for $x \geq 0$. The units of $X_{\mathbf{R}}$, are the nonzero multiplicative translations of $\mathbf{R}$ and may thus be identified with the multiplicative group $\mathbf{R}^{\#}$ of nonzero reals. Given $x, y>0$ in $\mathbf{R}$, the two isomorphisms of $\mathbf{Z x}$ upon $\mathbf{Z} y$ are the map $\langle y, x\rangle$ sending $n x$ to $n y$, and the map $-\langle y, x\rangle$ sending $n x$ to $-n y$. The complement of $\mathbf{R}^{\#}$ is a maximal ideal $\mathbf{M}$ consisting of the $D$-class of all isomorphisms between the nontrivial cyclic subgroups and the $D$-class $\{0\}$.

To further describe M, we define its rational core to be the bisimple subsemigroup $\mathbf{M}_{\mathcal{Q}}$ of all isomorphisms between nonzero cyclic subgroups of the additive group of rationals $Q$. Given positive rationals $u, v, x$ and $y$ with $\sigma, \tau \in \operatorname{sgn}$ (the sign group defined in Example 1.8), multiplication in $\mathbf{M}_{\mathbf{Q}}$ is described by:

$$
\sigma\langle v, u) \tau\langle y, x\rangle=\sigma \tau\langle v y /(u, y), u x /(u, y)\rangle
$$


where $(u, y)$ denotes the largest rational number $q$ for which both $u / q$ and $y / q$ are integers. $\mathbf{M}_{\mathbf{Q}}$ thus factors as the product of the group sgn with the subsemigroup $\mathbf{P}^{+}$of all positive cyclic isomorphisms, extending to all of $\mathbf{Q}^{+} \times \mathbf{Q}^{+}$(for $\mathbf{Q}^{+}$denoting the positive rationals) the multiplication defined for $\mathbf{P}$ in Example 1.8. If $\mathbf{B}^{\prime}$ denotes the semigroup defined on all $\mathbf{Z} \times \mathbf{Z}$ by extending the multiplication rule given for the bicyclic semigroup $\mathbf{B}$ in Example 1.8 , then a decomposition $\mathbf{P}^{+} \cong \Sigma^{\omega} \mathbf{B}^{\prime}$ is obtained by extending the isomorphism given for $\mathbf{P}$ in Example 1.8 to allow for negative exponents.

The maximal ideal, $\mathbf{M}$, is isomorphic to the matrix semigroup $\left(I \times \mathbf{M}_{\mathbf{Q}} \times I\right)^{0}$, for $I$ a set of coset representatives for the subgroup of positive rationals in the multiplicative group of positive reals, where nontrivial instances of multiplication are given by letting $(i, \alpha, j)(k, \beta, l)$ be $(i, \alpha \beta, l)$ if $j=k$, and 0 otherwise.

Finally, since extremal monomorphisms and extremal epimorphisms dualize each other, $X_{\mathrm{R}}$ is isomorphic with the dual monoid $X_{\mathrm{R}}^{*}$ arising in $\mathbf{E}^{x}(\mathbf{K})$.

\section{Symmetric monoids and inverse algebras}

Almost all important categories of mathematical objects are well endowed as categories. In particular, they are usually both complete and cocomplete. It follows that when one carefully examines the [dual] symmetric inverse monoids of their objects, one should expect to discover monoids that are correspondingly well endowed.

To see what occurs, recall first that an inverse monoid $S$ comes equipped with a natural partial ordering which is given by: $x \geq y$ in an inverse monoid $S$ if and only if any of the following equivalent conditions holds:
(i) $\exists e \in \mathrm{E}(S), y=e x$
(ii) $\exists f \in \mathbf{E}(S), y=x f$
(iii) $y=y y^{-1} x$
(iv) $y=x y^{-1} y$

where $\mathbf{E}(S)$ denotes the set of idempotents in $S$. (See [7, V.2].) Stated intuitively, $x \geq y$ asserts that $y$ is a restriction of $x$, which is the case when one examines the symmetric inverse monoid of a set $X$, or of any mathematical structure. (See [7, V.1.10].) An important property of this partial ordering is that it is compatible with both multiplication and inversion: $x \geq y$ and $u \geq v$ imply $x u \geq y v$ and $x^{-1} \geq y^{-1}$. Even more is true:

Lemma 2.1. Let $S$ be an inverse monoid. Then with respect to the natural partial ordering on $S$, both inversion and multiplication distribute over conditional infima. Thus if $\left\{x_{i} \mid i \in I\right\}$ for $I \neq \emptyset$ has an infimum, then so do $\left\{x_{i}^{-1} \mid i \in I\right\}$ and $\left\{u x_{i} v \mid i \in I\right\}$ for any $u, v \in S$ with

$$
\inf _{i \in I}\left\{x_{i}^{-1}\right\}=\left(\inf _{i \in I}\left\{x_{i}\right\}\right)^{-1} \text { and } \quad \inf _{i \in I}\left\{u x_{i} v\right\}=u\left(\inf _{i \in I}\left\{x_{i}\right\}\right) v
$$

That inversion distributes over conditional infima, as well as conditional suprema, is well-known. Less known, perhaps, has been the similar fact for multiplication, which appeared as an incidental result in a 1973 paper by Schein [17]. In order to exploit 
maximally the natural partial ordering in a systematic fashion, we shall require the following concept from [13]:

An inverse algebra is an inverse monoid which forms a meet semilattice under the natural partial ordering. More formally, an inverse algebra is an algebra $\left(S ; \bullet, \wedge,{ }^{-1}, 1\right)$ of type $\langle 2,2,1,0\rangle$ such that:

(i) the reduct $\left(S ; \bullet,{ }^{-1}, 1\right)$ is an inverse monoid (with the symbol $\bullet$ omitted when writing products);

(ii) the reduct $(A ; \wedge)$ is a meet semilattice;

(iii) the identities, $\left(a a^{-1} b\right) \wedge b=a a^{-1} b$ and $(a \wedge b)(a \wedge b)^{-1} b=(a \wedge b)$ hold.

Condition (iii) asserts that the natural partial order of both reducts agree. Clearly inverse algebras form a variety of algebras. An inverse algebra is complete if all nonempty subsets have infima. In what follows, the algebra under consideration is usually denoted by its underlying set $S$.

Perhaps the most striking contrast between inverse semigroups in general and inverse algebras is that of the role of the idempotents in the structure of an inverse algebra. To see this, let $x \in S$, for $S$ an inverse monoid. If an idempotent $f \in \mathbf{E}(S)$ exists such that

(i) $x \geq f$, that is, $x f=f=f x$, and

(ii) for any idempotent $e \in S$ such that $x \geq e$ it follows that $f \geq e$,

then $f$ is unique and is called the fixed point idempotent of $x$, to be denoted by $f[x]$. If $f[x]$ exists for all $x \in S$, then the induced unary operator $f: S \rightarrow \mathbf{E}(S)$ is called the fixed point operator of $S$ and the inverse monoid $S$ is said to "have fixed point idempotents." When this is the case, compatability of the partial order implies that for any $x, y \in S, f\left[x^{-1}\right]=f[x]$ and $f[x y] \geq f[x] f[y]$. We now recall the following fundamental result:

Theorem 2.2. ([13, Theorem 1.9]) An inverse monoid $S$ is an inverse algebra if and only if it has fixed point idempotents. When $S$ is an inverse algebra then also:

(i) For all $x, y \in S, x \wedge y=f\left[x y^{-1}\right] y=x f\left[x^{-1} y\right]$ and $f[x]=1 \wedge x$.

(ii) $S$ is complete if and only if $\mathbf{E}(S)$ forms a complete lattice, in which case infima of nonempty subsets of $S$ are given by:

$$
\inf _{\mathrm{i} \in \mathrm{I}} x_{\mathrm{i}}=\left(\inf _{\mathrm{i}, \mathrm{j} \in \mathrm{I}} f\left[x_{\mathrm{i}} x_{\mathrm{j}}^{-1}\right]\right) x_{\mathbf{k}} \text {, for any } k \in I \text {. }
$$

The pleasing fact that multiplication and inversion distribute over meets suggests a third approach to inverse algebras. To begin, a meet semilattice operation $\wedge$ defined on an inverse monoid is distributive if all inverse monoid operations distribute over $\wedge$. Question: Must distributive meets be natural? That is, does the presence of such a meet ensure (i) that the monoid is an inverse algebra and (ii) that the given meet coincides with the natural meet? To see that this need not be the case, consider a distributive 
lattice with a maximal element: $(D ; \vee, \wedge, 1)$. While $(D ; \wedge, 1)$ forms an inverse monoid, the distributive "meet" $\vee$ is not natural. Thus further conditions are needed to insure that the given meet is directed down the monoid. We pursue the matter further, beginning with a lemma.

Lemma 2.3. Let $S$ be an inverse monoid on which a distributive meet operation $\wedge$ is defined. Upon denoting the natural partial ordering by $\leq$ and the meet partial ordering by $\leq^{*}$, the following hold in $S$ :

(i) $x \leq^{*} y$ implies $u x v \leq *$ uyv and $x^{-1} \leq^{*} y^{-1}$.

(ii) $e, f \in \mathbf{E}(S)$ implies $e \wedge f \in \mathbf{E}(S)$ with $e \wedge f \leq *$ ef.

Moreover, the following are equivalent assertions:

(iii) $e \leq{ }^{*} 1$ for all $e \in \mathbf{E}(S)$.

(iv) $e \wedge f=e f$ for all $e, f \in \mathbf{E}(S)$.

(v) $x \leq y$ implies $x \leq^{*} y$ for all $x, y \in S$.

Proof. Distributivity plus the fact that $x \leq^{*} y$ means $x \wedge y=x$ yields (i). For (ii) first observe that $e \wedge f=e^{-1} \wedge f^{-1}=(e \wedge f)^{-1}$ and hence $(e \wedge f)^{3}=e \wedge f$. Distribution implies $(e \wedge f)^{2}=e \wedge f \wedge e f$ and so $(e \wedge f)^{2} \leq \leq^{*} e \wedge f, e f$. But then also, $e \wedge f=$ $(e \wedge f)^{3} \leq^{*}(e \wedge f)^{2}$ by (i) and so (ii) follows. To see the equivalence of (iii)-(v), observe first that (v) implies $e f \leq^{*} e \wedge f$ and hence (iv), which in turn implies (iii). So assume (iii). If $x \leq y$, then $x \wedge y=y x^{-1} x \wedge y=y\left(x^{-1} x \wedge 1\right)=y x^{-1} x=x$ and (v) follows.

Theorem 2.4. Let $S$ be an inverse monoid with a distributive meet operation $\wedge$ for which the induced partial ordering $\leq^{*}$ satisfies $e \leq^{*} 1$ for all $e \in \mathbf{E}(S)$. Then $S$ is an inverse algebra with the given meet $\wedge$ being the natural meet.

Proof. Pick $x \in S$ and set $f=1 \wedge x$. Distributivity implies that $f^{-1}=1 \wedge x^{-1}$ and also that $f=f f^{-1} f=1 \wedge x \wedge x^{-1} \wedge \ldots \leq^{*} 1 \wedge x^{-1}=f^{-1}$. Similarly $f^{-1} \leq * f$ and so $f=f^{-1}$. Thus $f=f^{3}$. But $f^{2}=1 \wedge x \wedge x \bar{x} \leq^{*} f$, so that $f^{3} \leq^{*} f^{2} \leq^{*} f$ and hence $f=f^{2}$. Now $f \leq^{*} x$ implies $f=f f \leq^{*} f x=\left(1 \wedge x^{-1}\right) x=x \wedge x x^{-1} \leq^{*} x \wedge 1=f$. Thus $f=f x$ and $f \leq x$ in the natural ordering. But if $e \in \mathbf{E}(S)$ and $e \leq x$, then by Lemma 2.3(v) $e \leq \leq^{*} x$ and so by Lemma 2.3(iv), $e=e \wedge 1 \wedge x=e \wedge f=e f$, that is, $e \leq f$. Thus $f$ is the fixed point idempotent of $x$, and $S$ is seen to form an inverse algebra. In fact $f[x]$ is given by the standard identity $f[x]=1 \wedge x$, but using the given meet. To complete the proof of the theorem, by Lemma 2.3(v), it suffices to show that $x \leq^{*} y$ implies $x \leq y$. So suppose $x \leq^{*} y$. By Lemma 2.3(i) we have $x=x x^{-1} x \leq^{*} y x^{-1} x$ so that $x=x \wedge y x^{-1} x=$ $\left(1 \wedge y x^{-1}\right) x=f\left[y x^{-1}\right] x$. But by Theorem 2.2(i), $f\left[y x^{-1}\right] x$ is the natural meet $x \wedge_{N} y$. Hence $x=x \wedge_{\mathrm{N}} y \leq y$.

Thus as algebras $\left(S ; \bullet, \wedge,,^{-1}, 1\right)$ of type $(2,2,1,0\rangle$, inverse algebras are determined by inverse monoid identities on $(S ; \bullet,-1,1)$, semilattice identities on $(S ; \wedge)$, the 
distributive identities, $u(x \wedge y) v=u x v \wedge u y v$ and $(x \wedge y)^{-1}=x^{-1} \wedge y^{-1}$, and the identity, $1 \wedge x x^{-1}=x x^{-1}$.

When is a symmetric inverse monoid an inverse algebra? When does a category have symmetric inverse algebras for each of its objects? When are such algebras complete? We begin with a lemma.

Lemma 2.5. Let $(\mathrm{M}, X)$ be a small monosetting. If $S=\mathrm{I}(\mathbf{M}, X)$ is the classifying monoid of the setting, then:

(i) $\mathbf{E}(S)=\{[\alpha, \alpha] \mid \alpha$ has codomain $X\}$

(ii) $\left[\alpha, \alpha^{\prime}\right] \geq\left[\beta, \beta^{\prime}\right]$ if and only if $(\exists \kappa \in \mathbf{M})\left(\beta, \beta^{\prime}\right)=\left(\alpha \kappa, \alpha^{\prime} \kappa\right)$

(iii) $\left[\alpha, \alpha^{\prime}\right] \geq[\beta, \beta] \in \mathbf{E}(S)$ if and only if there is a morphism $\delta \in \mathbf{M}$ such that $\alpha \delta=\beta=\alpha^{\prime} \delta$.

(iv) $\left[\alpha, \alpha^{\prime}\right]$ has a fixed point idempotent in $\mathrm{E}(S)$ if and only if the pair $\left(\alpha, \alpha^{\prime}\right)$ has an equalizer $\gamma$ in $\mathbf{M}$, in which case $f\left[\alpha, \alpha^{\prime}\right]=\left[\alpha \gamma, \alpha^{\prime} \gamma\right]$.

(v) $S$ is an inverse algebra if and only if $\mathbf{M}$ has equalizers.

(vi) $S$ is a complete inverse algebra if and only if $\mathbf{M}$ has equalizers and arbitrary nonempty intersections.

Proof. Assertion (i) is just Theorem 1.1(i) restated for convenience. To see (ii) let $\left[\alpha, \alpha^{\prime}\right] \in S$ and $[\varepsilon, \varepsilon] \in \mathbf{E}(S)$ be given, and let $\kappa$ and $\lambda$ be morphisms yielding intersection of the middle pair: $\alpha^{\prime} \kappa=\alpha^{\prime} \cap \varepsilon=\varepsilon \lambda$. We calculate:

$$
\left[\alpha, \alpha^{\prime}\right][\varepsilon, \varepsilon]=[\alpha \kappa, \varepsilon \lambda]=\left[\alpha \kappa, \alpha^{\prime} \kappa\right] .
$$

From the definition of the natural partial ordering, both (ii) and (iii) must follow. To see (iv), first suppose that $\gamma$ equalizes $\alpha$ and $\alpha^{\prime}$. Then by (iii) we have $\left[\alpha, \alpha^{\prime}\right] \geq\left[\alpha \gamma, \alpha^{\prime} \gamma\right] \in \mathbf{E}(S)$. Suppose next that $\left[\alpha, \alpha^{\prime}\right] \geq[\beta, \beta]$. By (iii) again, $\alpha \delta=\beta=\alpha^{\prime} \delta$ for some morphism $\delta$. Since $\gamma$ equalizes the pair $\left(\alpha, \alpha^{\prime}\right)$, there must be a factorization, $\delta=\gamma \kappa$. Thus by (ii), $\left[\alpha \gamma, \alpha^{\prime} \gamma\right] \geq\left[\alpha \gamma \kappa, \alpha^{\prime} \gamma \kappa\right]=[\beta, \beta]$, so that $\left[\alpha \gamma, \alpha^{\prime} \gamma\right]$ is the fixed point idempotent $f\left[\alpha, \alpha^{\prime}\right]$. Conversely, suppose that $S$ has fixed point idempotents, and let a parallel pair $\left(\alpha, \alpha^{\prime}\right)$ be given. Let $f\left[\alpha, \alpha^{\prime}\right]=[\varepsilon, \varepsilon]$. By (iii) a factorization $\alpha \gamma=\varepsilon=\alpha^{\prime} \gamma$ exists. We claim that $\gamma$ is the equalizer. For let $\alpha \delta=\alpha^{\prime} \delta$. By (iii), $\left[\alpha, \alpha^{\prime}\right] \geq$ $\left[\alpha \delta, \alpha^{\prime} \delta\right] \in \mathbf{E}(S)$. But since $[\varepsilon, \varepsilon]=f\left[\alpha, \alpha^{\prime}\right]$, we have $[\varepsilon, \varepsilon]=\left[\alpha \gamma, \alpha^{\prime} \gamma\right] \geq\left[\alpha \delta, \alpha^{\prime} \delta\right]$. Thus by (ii) there is a (necessarily unique) $\kappa$ such that $\alpha \delta=\alpha \gamma \kappa=\alpha^{\prime} \gamma \kappa=\alpha^{\prime} \delta$, so that $\gamma$ must indeed equalize $\left(\alpha, \alpha^{\prime}\right)$ and (iv) follows. Next, observe that by (iv) $S$ is an inverse algebra precisely when every pair $\left(\alpha, \alpha^{\prime}\right)$ with codomain $X$ has an equalizer in $\mathbf{M}$. Thus when $\mathbf{M}$ has equalizers, $S$ is an inverse algebra. For the converse, let $\left(\alpha, \alpha^{\prime}\right): A \rightarrow Y$ be any parallel pair in $M$. By the maximality of $X$, a morphism $\lambda: Y \rightarrow X$ must exist. If $S$ is an algebra, then the pair $\left(\lambda \alpha, \lambda \alpha^{\prime}\right)$ has an equalizer $\gamma: K \rightarrow A$. But since $\lambda$ is a monomorphism, $\gamma$ also equalizes $(\alpha, \alpha)$, so that (v) follows. Finally, (v), together with Theorem 2.2(ii), yields (vi). 
A category has [dual] symmetric inverse algebras if it has [dual] symmetric inverse monoids existing at each of its objects, with all such monoids being inverse algebras. When the algebras are also complete, the category is said to have complete [dual] symmetric inverse algebras. Immediate corollaries to the previous lemma are the following theorems and their duals.

Theorem 2.6. A well-powered category has symmetric inverse algebras if and only if (i) it has finite intersections and (ii) it has equalizers for parallel pairs of monomorphisms. In particular, any finitely complete, well-powered category has symmetric inverse algebras.

Theorem 2.7. A well-powered category $\mathbf{K}$ has symmetric inverse algebras that are complete if and only if (i) $\mathbf{K}$ has arbitrary, nonempty intersections and (ii) $\mathbf{K}$ has equalizers for all parallel pairs of monomorphisms. In particular, any complete, wellpowered category has complete symmetric inverse algebras.

Except for multiplicative semilattices, an E-unitary inverse monoid cannot be an inverse algebra since no non-idempotent in such a monoid is bounded below by an idempotent. This observation enables us to determine when a category has E-unitary symmetric inverse monoids.

Theorem 2.8. A well-powered category $\mathbf{K}$ with finite intersections has E-unitary symmetric inverse monoids if and only if its subcategory $\mathbf{M ( K )}$ of all monomorphisms is cancellative. In particular, any well-powered cancellative category with finite intersections has E-unitary symmetric inverse monoids.

Proof. To assert that no non-idempotent in any monoid $I_{\mathrm{X}}$ is bounded below by an idempotent is, by Lemma 2.5 (iii), to assert that for any parallel pair $\left(\alpha, \alpha^{\prime}\right)$ in $\mathbf{M}(\mathbf{K})$, $\alpha \kappa=\alpha^{\prime} \kappa$ for some $\kappa$ in $\mathbf{M}(\mathbf{K})$ if and only if $\alpha=\alpha^{\prime}$, which in turn is to assert that $\mathbf{M}(\mathbf{K})$ is right cancellative. Since monocategories are left cancellative by definition, the result follows.

There is more. Recall that an inverse monoid $S$ has conditional joins if every pair of elements $x, y \in S$ bounded above in $(S, \geq)$, has a least upper bound, $x \vee y$ called the natural join of $x$ and $y$. An inverse algebra with conditional joins is called finitely complete (the finitary version of complete inverse algebras which possess conditional suprema). Having conditional joins is a partial dual to having unconditional meets (in an inverse algebra), but in general it is the best one can expect. Both inverse algebras and E-unitary inverse monoids (but not arbitrary inverse monoids!) have conditional joins whenever their idempotents form a lattice, since $x \vee y=\left(x x^{-1} \vee y y^{-1}\right) u$ for any upper bound $u$ of $x$ and $y$.

Theorem 2.9. A well-powered and co-well-powered, cancellative category with both intersections and co-intersections has E-unitary [dual] symmetric inverse monoids which have conditional joins. 
Proof. In view of Theorem 1.1(i), we need to show that any object $X$ in such a category $K$ has a lattice of subobjects. So let $\alpha: A \rightarrow X$ and $\beta: B \rightarrow X$ be a pair of representative subobject morphisms. The meet $\alpha \wedge \beta$ is given by the intersection morphism $\alpha \kappa=\beta \lambda$, where $\kappa: \mathrm{I} \rightarrow \mathrm{A}$ and $\lambda: \mathrm{I} \rightarrow \mathrm{B}$ provide the intersection. The join $\alpha \vee \beta$ is the morphism $\eta: J \rightarrow X$ induced from the co-intersection in $\mathbf{K}$ of the pair $\kappa$ and $\lambda$ from I. Indeed there exist morphisms $\mu: \mathrm{A} \rightarrow \mathrm{J}$ and $v: \mathrm{B} \rightarrow \mathrm{J}$ creating the pushout. Since $\alpha \kappa=\beta \lambda$, the pair $(\alpha: \mathrm{A} \rightarrow \mathrm{X}, \beta: \mathbf{B} \rightarrow \mathrm{X})$ factors through the pair $(\mu: \mathrm{A} \rightarrow \mathrm{J}$, $v: \mathrm{B} \rightarrow \mathrm{J}$ ), with $\eta$ being the common complementary factor. (Draw the diagram.) It is easily seen that $\eta$ factors through any morphism $\theta: \mathrm{K} \rightarrow \mathrm{X}$ which is a common upper bound for $\alpha$ and $\beta$.

The introduction of conditional joins into the discussion reveals a linear hierarchy of algebraic structures: inverse monoids, inverse algebras, finitely complete inverse algebras, and complete inverse algebras. Our perspective thus leads us to ask: Under what conditions will a category $\mathbf{K}$ possess finitely complete symmetric inverse algebras? If we relax the assumption of arbitrary intersections, then finite completeness will not suffice. Indeed let $(S, \geq)$ be a meet semilattice, which is not a lattice, but has a supremum, $\Omega$. Then the dual category, $(S, \geq)^{\text {op }}$ is finitely complete, but the subobject semilattice at $\Omega$ is not a lattice. Will finite completeness and finite cocompleteness combined yield both symmetric and dual symmetric inverse algebras which are finitely complete? Yes, if we assume that $\mathbf{K}$ also has unique epi-mono factorizations so that every morphism factors into the composite $\alpha \beta$ of an epimorphism $\beta$, followed by a monomorphism $\alpha$, with all other such factorizations $\alpha^{\prime} \beta^{\prime}$ being of the form $\beta^{\prime}=\mu \beta$ and $\alpha^{\prime}=\alpha \mu^{-1}$ for some isomorphism $\mu$.

Theorem 2.10. If category $\mathbf{K}$ is both finitely complete and finitely cocomplete and possesses unique epi-mono factorizations, then symmetric inverse algebras and dual symmetric inverse algebras for all objects in $\mathbf{K}$ are finitely complete.

Proof. If category $\mathbf{K}$ has finite coproducts and unique epi-mono factorizations, then all subobject classes of objects in $\mathbf{K}$ have finite joins. For given subobjects $\alpha: A \rightarrow X$ and $\beta: B \rightarrow X$, a morphism $\theta: A+B \rightarrow X$ is determined such that $\alpha=\theta \eta_{\mathrm{A}}$ and $\beta=\theta \eta_{\mathrm{B}}$ where $\eta_{\mathrm{A}}$ and $\eta_{\mathrm{B}}$ are coproduct injections of $A$ and $B$ into $A+B$. The epi-mono factorization, $\theta=\gamma \varepsilon$, yields the subobject join setup: $\gamma: C \rightarrow X, \varepsilon \eta_{\mathrm{A}}: A \rightarrow C$ and $\varepsilon_{\mathrm{B}} \eta: B \rightarrow C$. The theorem follows from the implication and its dual.

Two very important classes of categories exist which satisfy the above conditions, but for which [co]-completeness is neither assumed nor implied.

Corollary 2.11. If $\mathrm{K}$ is an Abelian category, then it has finitely complete [dual] symmetric inverse algebras.

Corollary 2.12. If $\mathrm{K}$ is a topos, then it has finitely complete [dual] symmetric inverse algebras. 
For numerous familiar categories, epi-mono factorizations need not be unique, unless added conditions are required of the factorizations. These include the categories of rings and of [Hausdorff] topological spaces, both of which have complete [dual] symmetric inverse algebras anyway, as they are both complete and cocomplete categories. These categories do have unique factorizations if we replace "epi" by "extremal epi" [or else "mono" by "extremal mono"], in which case the [dual] argument in the above theorem still holds.

Like inverse monoids and inverse algebras, finitely complete inverse algebras also form $a$ variety. The join $\vee$ on $\mathrm{E}(S)$ can be extended to a pseudo-join operation + on $S$ defined by $x+y=x x^{-1} \vee y y^{-1}$, which also has $\mathbf{E}(S)$ as its set of idempotents. The standard lattice identities holding on $\mathbf{E}(S)$ can be transformed into identities involving + , thus determining a variety of enriched algebras $\left(S, \bullet, \wedge,,^{-1}, 1\right)$ of type $(2,2,2,1,0)$. For instance, $e \vee f=f \vee e$ and $e \wedge(e \vee f)=e$ on $\mathbf{E}(S)$ induce $x+y=y+x$ and $x x^{-1}(x+y)=x x^{-1}$ on $S$. Conversely, given any inverse monoid $S$ upon which an operator + is defined satisfying all transformed identities plus $x+x=x x^{-1}$, then $(E(S), \wedge,+)$, where $e \wedge f=e f$, is a lattice. If $S$ is an inverse algebra, then by previous remarks $S$ is also finitely complete. A variety of finitely complete inverse algebras is thus determined.

Complete inverse algebras, on the other hand, do not form a variety of algebras. Nonetheless, free complete inverse algebras exist. For given any set $X$, if $F(X)$ is the free inverse monoid on $X$, then the free complete inverse algebra on $X$ is given as the full coset algebra $\mathbf{K}(F(X))$ together with the composite inclusion: $X \rightarrow F(X) \rightarrow$ $\mathbf{K}(F(X))$. (See [13] for details.) Complete inverse algebras thus behave like ordinary algebras in a variety, as well as like infinitary structures such as sup-semilattices and compact Hausdorff spaces. In this regard they are unlike complete lattices or complete Boolean algebras which in general fail to have free algebras. (See Gaifman [4] and Hales [6], or Johnstone [8].)

A category has symmetric inverse semilattices if it has symmetric inverse monoids, all of which are semilattices. In this regard, recall that a category is a quasi-order if all of its morphism sets $\operatorname{hom}(A, B)$ have a most one morphism. An immediate corollary of Theorem 1.1(i) is:

Lemma 2.13. A well-powered category $\mathbf{K}$ has symmetric inverse semilattices if and only if $\mathbf{K}$ has finite intersections and the monocategory $\mathbf{M}(\mathbf{K})$ of all monomorphisms in $\mathbf{K}$ is a quasi-order.

Before presenting our next theorem, we state two more lemmas.

Lemma 2.14. In any monocategory, morphisms arising in any factorization of an isomorphism are also isomorphisms (so that isomorphisms are closed under factorization).

Lemma 2.15. Let $\mathbf{K}$ be a category whose isomorphisms are closed under factorization. Then for any object $X$ of $\mathbf{K}$ the power $X \times X$ exists if and only if for all objects $U$ in $\mathrm{K}$, the morphism class $\operatorname{hom}(U, X)$ has at most one morphism, in which case $X \times X=X$ 
with the power projections $\pi_{\mathrm{i}}: X \rightarrow X$ for $i=1,2$ being the identity morphisms. Dual remarks hold for the copower, $X+X$.

Theorem 2.16. Given a category $\mathrm{K}$ with finite intersections, the following are equivalent.

(i) The monocategory $\mathbf{M}(\mathbf{K})$ has powers $X \times X$.

(ii) The monocategory $\mathbf{M}(\mathbf{K})$ has copowers $A+A$.

(iii) The monocategory $\mathbf{M}(\mathbf{K})$ is a quasi-order.

(iv) All objects of $\mathbf{K}$ are terminal in their monosettings.

(v) All objects of $\mathbf{K}$ have symmetric inverse semilattices.

(vi) All objects of $\mathbf{K}$ have finitely complete monosettings.

Proof. By the previous three results, conditions (i)-(v) are equivalent. Clearly (vi) implies (i). Conversely, given (i)-(v), any monosetting in $\mathbf{K}$ is finitely complete, since it is a quasi-order with finite intersections.

Theorem $2.16 \mathrm{can}$, in part, be generalized. To begin, by the tail $\tau(S)$ of an inverse semigroup $S$ is meant the set $\left\{x \in S \mid x S x^{-1} \subseteq \mathbf{E}(S)\right\}$, or equivalently, the set $\left\{x \in S \mid x x^{-1} S x x^{-1} \subseteq \mathbf{E}(S)\right\}$. When $S=\tau(S)$, then $S$ is a subdirect product of Brandt semigroups having trivial subgroups, and conversely. (See [15, II.4, and in particular, Exercise II.4.8(ix)].) In general $\tau(\tau(S))=\tau(S)$ and when nonempty, $\pi(S)$ forms an ideal.

An object $A$ in a category $\mathbf{K}$ is subterminal if for all objects $B$ in $\mathbf{K}$, the morphism set $\operatorname{hom}(B, A)$ has at most one element. Subobjects of subterminal objects are clearly subterminal, and if $\mathbf{K}$ has finite intersections, then the class of subterminal subobjects of any object $X$ in $\mathbf{K}$ is closed under pairwise intersection. By Lemma 2.5, subterminal objects in a monosetting are precisely those objects $A$ for which the power $A \times A$ exists in the given monosetting. The effect of subterminal objects upon the structure of the classifying monoid is as follows.

Proposition 2.17. Let the inverse monoid $S$ classify a small monosetting $(M, X)$. Then $\tau(S)=\left\{\left[\alpha, \alpha^{\prime}\right] \mid \alpha\right.$ and $\alpha^{\prime}$ share a subterminal domain $\}$.

Proof. Let $\left(\alpha, \alpha^{\prime}\right): Y \rightarrow X$ be a parallel pair of morphisms in $\mathbf{M}$ and let $\mathbf{M}_{Y}$ denote the monosetting of $Y$ in $M$. In general, $I\left(M_{Y}, Y\right) \cong[\alpha, \alpha] S[\alpha, \alpha]$ under the function $\left[\beta, \beta^{\prime}\right] \rightarrow\left[\alpha \beta, \alpha \beta^{\prime}\right]$. But since $\left[\alpha, \alpha^{\prime}\right] S\left[\alpha^{\prime}, \alpha\right]=[\alpha, \alpha] S[\alpha, \alpha]$, it follows that $\left[\alpha, \alpha^{\prime}\right] S\left[\alpha^{\prime}, \alpha\right] \subseteq \mathbf{E}(S)$ if and only if $Y$ is terminal in $\mathbf{M}_{Y}$, that is to say, $Y$ is subterminal in $\mathbf{M}$.

Tails appear in another context. But first, the (idempotent) cover of an element $x$ in an inverse monoid $S$ is the least idempotent $c$ such that $c x=x c=x$. If it exists, $c$ yields 
the smallest principal submonoid $c S c$ of $S$ containing $x$. When covers exist for all elements, a covering operator $c[x]$ is defined and $S$ is said to have covers. This always occurs when $S$ has conditional joins, since then $c[x]=x x^{-1} \vee x^{-1} x$. Having covers is thus a weakening of having conditional joins. The operator $c[x]$ also dualizes of the operator $f[x]$, provided one changes the partial ordering from the natural one to that given by annihilation: $x \geq_{\mathrm{A}} y$ if and only if either $x=y$ or $x y=y=y x$. An inverse monoid $S$ has maximal covers if $c[x]=1$ holds for all non-idempotents $x$. (As always, $c[e]=e$ for any idempotent $e$.)

Theorem 2.18. An inverse monoid $S$ has maximal idempotent covers if and only if the complement $M$ in $S$ of the group of units $G$ is an ideal of $S$ lying inside the tail $\tau(S)$ of $S$. Moreover, an inverse monoid $S$ having maximal covers forms an inverse algebra if and only if each unit $u \in G$ has a fixed point idempotent; in particular, $S$ in an inverse algebra if $G=\{1\}$.

Proof. To begin, let $S$ have maximal covers and let $u v=1$ in $S$. If $v u \neq 1$, then $v u$ is an idempotent distinct from 1 , and $v^{2} u$ is a nonidempotent for which $v u\left(v^{2} u\right)=v^{2} u=\left(v^{2} u\right) v u$, contradicting the maximality assumption for $v^{2} u$. Thus in $S$, $u v=1$ if and only if $v u=1$, and this insures that the complement $M$ of $G$ is an ideal of $S$. If $x \in M$, then $x x^{-1} \neq 1$ in $\mathbf{E}(S)$ and for all $y \in S:\left(x x^{-1}\right) x y x^{-1}=x y x^{-1}=$ $x y x^{-1}\left(x x^{-1}\right)$. By maximality, $x y x^{-1} \in \mathbf{E}(S)$, so that $M \subseteq \tau(S)$ must follow. Conversely, suppose that $M=S-G$ is an ideal of $S$ such that $M \subseteq \tau(S)$. Clearly $c[u]=1$ for all $u \in G$. Suppose that $x \in M$ is not idempotent, but that $e x=x=x e$ for $e$ an idempotent. Then $e=1$, since otherwise $e \in \tau(S)$ in which case $x=e x e \in \mathrm{E}(S)$. Thus $c[x]=1$ for all non-idempotent elements $x$, so that $S$ must have maximal covers. In general, for any inverse monoid $S$, if $x \in \tau(S)$, then $f[x]$ exists and is given by $x^{2}$. Indeed the equation $x^{2}=x^{3}$ holds in any Brandt semigroup with trivial subgroups, and thus must hold in $\tau(S)$ which is a subdirect product of such semigroups, so that $f[x]=x^{2}$. It follows that for inverse monoids having maximal idempotent covers, the presence of fixed point idempotents is reduced to what happens in the case of the units, and so the second biconditional assertion is verified.

\section{Free categories}

Let $\mathbf{G}$ be a directed graph (possibly infinite) and let $\mathbf{G}^{*}$ denote the small category freely generated from $\mathbf{G}$. In light of Theorem 1.3, it is natural to ask what conditions on $\mathbf{G}$ insure that $\mathbf{G}^{*}$ will have finite intersections? We begin with some observations: (i) $\mathbf{G}^{*}$ is well-powered; (ii) the composition in $\mathbf{G}^{*}$ is cancellative so that all morphisms are both monic and epic; (iii) only the identity morphisms are isomorphisms; (iv) every nonidentity morphism in $\mathbf{G}^{*}$ factors uniquely into a product of consecutive edges in G. (Edges $\beta$ and $\alpha$, in that order, are consecutive if $\alpha$ enters the vertex that $\beta$ is leaving, so that " $\beta \alpha$ " denotes a directed path of length 2 when read from right to left. Although our reading of paths reverses the standard reading in graph theory, it agrees with the composition of morphisms in the induced free category $\mathbf{G}^{*}$.) 
Lemma 3.1. If the free category $\mathbf{G}^{*}$ on a directed graph $\mathbf{G}$ has finite intersections, then every vertex in $\mathrm{G}$ has at most one entering edge.

Proof. Suppose that edges $\alpha$ and $\beta$ in $\mathbf{G}$ enter a common vertex $v$, with $\alpha$ and $\beta$ leaving the respective vertices $a$ and $b$. If an intersection $\alpha \cap \beta$ exists in $\mathbf{G}^{*}$ then there is a vertex $u$ and morphisms, $\gamma^{*}: u \rightarrow a$ and $\delta^{*}: u \rightarrow b$, such that $\alpha \gamma^{*}=\beta \delta^{*}$. By unique factorization of the morphisms into consecutive edges, $\alpha=\beta$ follows.

The condition on $\mathbf{G}$ stated in the lemma is also sufficient to insure that $\mathbf{G}^{*}$ has finite intersections. To see this, consider the following four cases, each involving a graph with a terminal vertex labelled $u_{0}$.

(i) $\mathbf{L}_{\mathbf{n}}$ with $\mathbf{n} \geq 0$ is the linear graph,

$$
\mathbf{L}_{\mathbf{n}}: u_{0} \leftarrow u_{1} \leftarrow u_{2} \leftarrow \cdots \leftarrow u_{\mathrm{n}-1} \leftarrow u_{\mathbf{q}} .
$$

(ii) $\mathbf{L}_{\infty}$ is the infinite linear graph,

$$
\mathbf{L}_{\infty}: u_{0} \leftarrow u_{1} \leftarrow u_{2} \leftarrow \cdots \leftarrow u_{\mathrm{n}} \leftarrow \cdots
$$

(iii) $\Gamma_{\mathbf{d}}$ with $\mathbf{d} \geq 1$ is the circuit (cyclicly directed graph) on $\mathbf{d}$ vertices,

$$
\Gamma_{\mathrm{d}}: u_{0} \leftarrow u_{1} \leftarrow u_{2} \leftarrow \cdots \leftarrow u_{\mathrm{d}-2} \leftarrow u_{\mathrm{d}-1} \leftarrow u_{0} \text {. }
$$

(iv) The splice $\mathbf{L}_{\mathbf{b}} \Gamma_{\mathbf{d}}$ with both $\mathbf{d}, \mathbf{n} \geq 1$ and described as follows,

$$
\mathbf{L}_{\mathrm{b}} \Gamma_{\mathrm{d}}: u_{0} \leftarrow u_{1} \leftarrow \cdots \leftarrow u_{\mathrm{n}-1} \leftarrow u_{\mathrm{n}} \leftarrow u_{\mathrm{n}+1} \leftarrow \cdots \leftarrow u_{\mathrm{n}+\mathrm{d}-1} \leftarrow u_{\mathrm{n}} \text {. }
$$

Note that $\mathbf{L}_{0}$ consists of a single vertex, while $\Gamma_{1}$ consists of a vertex with a single directed loop.

Notation. Given vertex $v$ in a directed graph $\mathbf{G}$, then $\mathbf{G}^{v}$ denotes the subgraph consisting of edges arising in some directed path terminating at $v$, along with all incident vertices.

Lemma 3.2. All four types of directed graphs listed above satisfy the condition that each of its vertices has at most one entering edge. Conversely, let $\mathbf{G}$ be a directed graph satisfying this condition. Then for any vertex $v$ in $\mathbf{G}$, the subgraph $\mathbf{G}^{v}$ is isomorphic to exactly one of the types of graphs listed above.

Proof. The first assertion is clear. For the converse, if the condition holds, then given any vertex $v$, either there is no edge entering $v$, or else $v$ has a unique predecessor vertex $u$, the starting vertex of the edge entering $v$. We can thus start at $v$, back up to the predecessor $u$, and then repeat the process. We have the following cases, each corresponding to the four types of graphs listed above: (i) After $\mathbf{n}$ steps we come to a vertex without a predecessor, and the process stops. (ii) The process never stops. No vertex upon being first encountered is ever encountered again. (iii) After d steps the 
starting vertex $v$ is encountered again, and the process repeats itself. (iv) After $\mathbf{n}+\mathbf{d}$ steps a subsequent vertex $u$, first encountered after $\mathbf{n}$ steps, is re-encountered. The process, beginning with the first encounter of $u$, is now like the third case.

Lemma 3.3. All four types of graphs listed above freely generate categories having finite intersections.

Proof. Due to the unique factorization of morphisms into edges, the following situation holds in all four cases: if morphisms $\alpha$ and $\beta$ are of the same length and have a common codomain vertex, then $\alpha=\beta=\alpha \cap \beta$. (The length of a morphism is the number of edges appearing in its unique factorization into consecutive edges.) Otherwise, again let $\alpha$ and $\beta$ have a common codomain, but suppose $\alpha$ is of greater length. By unique factorization again, a unique morphism $\gamma$ exists such that $\alpha=\beta \gamma$. The intersection $\alpha \cap \beta$ is now given by the pair $(l, \gamma)$ where $l$ is the identity on the domain vertex of $\alpha$.

Theorem 3.4. The free category $\mathbf{G}^{*}$ on a directed graph $\mathbf{G}$ has finite intersections (and thus symmetric inverse monoids for each vertex) if and only if no vertex $v$ of $\mathbf{G}$ has more than one edge coming into it.

Proof. Let $v$ be a vertex in $\mathbf{G}$. As an object in $\mathbf{G}^{*}$, the monocontext of $v$ is precisely $\mathbf{G}^{* v}=\mathbf{G}^{v *}$. The theorem now follows from the previous three lemmas.

What precisely are the possibilities for G? Clearly it suffices to describe the possibilities when $\mathbf{G}$ is connected as a graph (without considering the direction of its edges). To do so, we shall need the following concepts.

To begin, recall that a rooted tree is a directed graph $\mathbf{T}$ with a distinguished vertex $r$ (the root) such that given any vertex $v$, there is a unique directed path in $\mathbf{T}$ from $r$ to $v$. Given an undirected tree $\mathbf{T}$ and a vertex $r$ of $\mathbf{T}$, there is a unique way to direct the edges of $\mathbf{T}$ so that it becomes a rooted tree at $r$. We allow $\mathbf{T}$ to be countably infinite.

A directed tree is a directed graph $\mathbf{T}$ which can be expressed as an infinite, properly ascending, union of subgraphs,

$$
T=U T_{n}, \quad T_{1} \subset T_{2} \subset T_{3} \subset \ldots \subset T_{n} \subset \ldots,
$$

each forming a rooted tree which is an "ideal" of $T$ : given vertex $v_{n} \in T_{n}$ and a directed path in $T$ from $v_{\mathrm{a}}$ to a vertex $x \in T$, then both $x$ and the path lie in $T_{n}$.

Observe that both rooted trees and directed trees have their vertices partially ordered upon setting $u \leq v$ if and only if there is a directed path from $u$ to $v$. As such the vertices form a meet semilattice. The tree is rooted if and only if there is a minimal element 0 (given by the root).

Recall that a cycle of order $\mathrm{n}$ is any directed graph isomorphic with $\Gamma_{\mathbf{n}}$ for some $\mathbf{n} \geq 1$. A cycle of trees is the directed graph obtained by joining a finite number of disjoint rooted trees $T_{1}, \ldots, T_{n}$ by inserting the same number of directed edges at the roots $r_{1}, \ldots, r_{\mathrm{b}}$ to form a cycle of the same order: say $r_{1} \leftarrow r_{2} \leftarrow \ldots \leftarrow r_{\mathrm{a}} \leftarrow r_{1}$. (A cycle 
is thus understood to be a limiting case of a cycle of trees.) The following result is now clear:

Theorem 3.5. A free category $\mathbf{G}^{*}$ on a connected directed graph $\mathbf{G}$ has symmetric inverse monoids if and only if $\mathbf{G}$ is isomorphic with one of the following three types of graphs: a rooted tree, a directed tree, or a cycle of trees. Moreover, if $\mathbf{G}$ is a rooted tree, then every subgraph $\mathbf{G}^{0}$ has the form $\mathbf{L}_{\mathbf{b}}$ for $\mathbf{n}=\mathbf{n}(v)$. If $\mathbf{G}$ is a directed tree, then every subgraph $\mathbf{G}^{v}$ has the form $\mathbf{L}_{\infty}$. Finally if $\mathbf{G}$ is a cycle of trees, then every $\mathbf{G}^{v}$ has the form $\Gamma_{\mathrm{n}}$ or $\mathbf{L}_{\mathrm{m}} \Gamma_{\mathrm{a}}$ for $\mathbf{n}$ fixed, but $\mathbf{m}$ dependent on the vertex $v$.

By the dual of Theorem 3.4 , every object (vertex) in a free category $\mathbf{G}^{*}$ has finite co-intersections if and only if every vertex in the graph $\mathbf{G}$ has at most one edge leaving it. From the duals of Theorems 3.4 and 3.5 we obtain:

Theorem 3.6. The free category $\mathbf{G}^{*}$ on a directed graph $\mathbf{G}$ has both symmetric inverse monoids and dual symmetric inverse monoids if and only if every vertex $v$ of $\mathbf{G}$ has at most one edge entering it and at most one edge leaving it. To within isomorphism of directed graphs, the only possible connected graphs satisfying these conditions are the following:

(i) $\mathbf{L}_{\mathbf{n}}$ for $\mathbf{n} \geq 0$;

(ii) $\mathbf{L}_{\infty}$;

(iii) the oppositely directed graph $\mathbf{L}_{\infty}^{\mathrm{op}}$;

(iv) the splice of $\mathbf{L}_{\infty} \mathbf{L}_{\infty}^{o p}$ given by identifying $u_{0}$ in both graphs;

(v) the various $\Gamma_{\mathrm{d}}$ for $\mathrm{d} \geq 1$.

(The graph of (iv) is equivalent to the directed graph $\mathbf{Z}$ having the integers as vertices, and the edges given by the successor relation, $n \rightarrow n+1$.)

What kinds of monoids can arise as symmetric inverse monoids in a free category $\mathbf{G}^{*}$ having finite intersections?

Theorem 3.7. The only types of inverse monoids arising as [dual] symmetric inverse monoids in free categories are finite chains and $\omega$-semigroups with trivial subgroups. For free categories with both finite intersections and finite co-intersections, the only types of [dual] symmetric inverse monoids which can arise are finite chains, $\omega$-semilattices, and simple $\omega$-semigroups with trivial subgroups.

Proof. We need only consider the case where $\mathbf{G}$ is connected.

Case 1: $\mathbf{G}$ is a rooted tree. At each vertex $v, \mathbf{G}^{v}$ is a linear graph of the form $\mathbf{L}_{n}$, say $v=u_{0} \rightarrow u_{2} \rightarrow \cdots \rightarrow u_{\mathrm{v}-1} \rightarrow u_{\mathrm{n}}$. Then for each $u_{\mathrm{k}}$ there is a unique morphism $\alpha_{\mathrm{k}}$ in $\mathbf{G}^{0 *}$ from $u_{\mathrm{k}}$ to $u_{0}$. The monoid $I_{v}$ is thus the totally ordered semilattice of length $\mathbf{n}$.

$$
1=\left[\alpha_{0}, \alpha_{0}\right]>\left[\alpha_{1}, \alpha_{1}\right]>\left[\alpha_{2}, \alpha_{2}\right]>\cdots>\left[\alpha_{1}, \alpha_{\mathrm{a}}\right]
$$


Case 2: $\mathbf{G}$ is a directed tree, then $\mathbf{G}^{0}$ is an infinite linear graph of the form $\mathbf{L}_{\infty}$ for each vertex $v$. By reasoning similar to that in the first case, it follows that in this case $I_{v}$ is the $\omega$-semilattice,

$$
1=\left[\alpha_{0}, \alpha_{0}\right]>\left[\alpha_{1}, \alpha_{1}\right]>\left[\alpha_{2}, \alpha_{2}\right]>\cdots>\left[\alpha_{n}, \alpha_{n}\right]>\cdots
$$

Case 3: $\mathbf{G}$ is a cycle of rooted trees. At each vertex $v$, the graph $\mathbf{G}^{v}$ is isomorphic to either of a cycle $\Gamma_{d}$ or a splice $L_{d} \Gamma_{d}$ with $n \geq 1$. We consider the latter, and calculate $I_{u}$ for $u=u_{0}$ in the category $\mathbf{L}_{\mathrm{n}} \Gamma_{\mathrm{d}}^{*}$. Using the notation for $\mathbf{L}_{\mathrm{a}} \Gamma_{\mathrm{d}}$ the following hold in $\mathbf{L}_{\mathrm{b}} \Gamma_{\mathrm{d}}^{*}$. For $\mathbf{k}<\mathbf{n}$, a unique morphism $\alpha_{\mathrm{k}}: u_{\mathbf{k}} \rightarrow u_{0}$ exists; for $\mathbf{k} \geq \mathbf{n}$, there exist infinitely many morphisms from $u_{k}$ to $u_{0}$, to be denoted $\alpha_{k}, \alpha_{k+d}, \alpha_{k+2 d}, \ldots, \alpha_{k+p d}, \ldots$ where in all cases the subscript is given by the length of the morphism. The elements of $I_{u}$ thus consists of: (i) an infinitely descending chain of idempotents $\left[\alpha_{n}, \alpha_{n}\right], n \geq 0$, as in case 2; (ii) elements of the form $\left[\alpha_{i}, \alpha_{j}\right]$ where $i, j \geq n$ and $(i-j)$ is a multiple of $d$. Intersection in $\mathbf{L}_{\mathrm{d}} \Gamma_{\mathrm{d}}^{*}$ is given by $\alpha_{i} \cap \alpha_{j}=\alpha_{\max (\mathrm{i}, j)}$ with the pair of factors $(\kappa, \lambda)$ being the morphisms $\kappa$ of length $\mathrm{m}-\mathrm{i}$ from $u_{\mathrm{m}}$ to $u_{\mathrm{i}}$ and $\lambda$ of length $\mathrm{m}-\mathrm{j}$ from $u_{\mathrm{m}}$ to $u_{\mathrm{j}}$ respectively. Thus multiplication is a restriction of that encountered in the bicyclic semigroup:

$$
\left[\alpha_{i}, \alpha_{j}\right]\left[\alpha_{k}, \alpha_{1}\right]=\left[\alpha_{i+k-\min (j, k)}, \alpha_{j+1-\max (j, k)}\right]
$$

One thus has an $\omega$-semigroup consisting of a chain of $\mathbf{n}$ idempotents, from $\left[\alpha_{0}, \alpha_{0}\right]$ down to $\left[\alpha_{0-1}, \alpha_{0-1}\right]$, followed by a simple ideal $B_{d}$ characterized to within isomorphism as a simple $\omega$-semigroup having $\mathrm{d} D$-classes and trivial subgroups.

Such semigroups have been characterized separately by Munn [14] and Kochin [9]. (See also [7, Sections V.6 and V.7], or [15, Chapter XI].)

Acknowledgement. The author expresses his appreciation to Des FitzGerald for bringing to his attention numerous suggestions for the improvement of a preliminary draft of this paper. In particular, the author is indebted to him for simplifications in the proof of Theorem 2.4. The author is also indebted to the referee for several helpful suggestions regarding the organization of this paper.

\section{REFERENCES}

1. J. Ademek, H. Herrlich and G. E. Strecker, Abstract and Concrete Categories (Wiley Interscience, New York, 1990).

2. M. BARR and C. Wells, Toposes, Triples and Theories (Springer-Verlag, New York, 1985).

3. A. H. Clifford, A class of d-simple semigroups, Amer. J. Math. 15 (1953), 541-556.

4. H. GaIfMAN, Infinite Boolean polynomials, I, Fund. Math. 54 (1964), 229-250.

5. P. A. Grillet, Semigroups, An Introduction to the Structure Theory (Marcel Dekker, New York, 1995).

6. A. W. Hales, On the non-existence of free complete Boolean algebras, Fund. Math. 54 (1964), 45-66. 
7. J. M. HowIE, An Introduction to Semigroup Theory (Academic Press, London, 1976).

8. P. T. Johnstone, Stone Spaces (Cambridge University Press, Cambridge, 1982).

9. B. P. Kochin, The structure of inverse ideal-simple $\omega$-semigroups, Vestnik Leningrad. Univ. 23 (1968), 41-50 (in Russian).

10. M. V. Lawson, The geometric theory of inverse semigroups, I, J. Pure Appl. Algebra 67 (1990), 151-177.

11. J. E. LEECH, The D-category of a monoid, Semigroup Forum 34 (1986), 89-116.

12. J. E. LEECH, Constructing inverse monoids from small categories, Semigroup Forum 36 (1987), 89-116.

13. J. E. LeECH, Inverse monoids with a natural semilattice ordering, Proc. London Math. Soc. (3) 70 (1995), 146-182.

14. W. D. MunN, Regular $\omega$-semigroups, Glasgow Math. J. 9 (1968), 44-66.

15. M. Petrich, Inverse Semigroups (John Wiley and Sons, New York, 1984).

16. L. S. Pontr Yagin, Topological Groups, Second Edition (Gordon and Breach, New York, 1966).

17. B. M. SCheIN, Completions, translational hulls and ideal extensions of inverse semigroups, Czechoslovak Math. J. 23 (1973), 575-610.

Department of Mathematics

WESTMONT COLLEGE

955 la Paz Road

Santa Barbara, California

93108-1099

U.S.A.

E-mail address: leech@westmont.edu 